\title{
Optimizing drug delivery using non-uniform magnetic fields: a numerical study
}

\author{
J.W. Haverkort ${ }^{1}$ and S. Kenjereš ${ }^{1,2}$ \\ ${ }^{1}$ Delft University of Technology / Multi-Scale Physics, Delft, The Netherlands \\ ${ }^{2}$ J. M. Burgerscentre for Fluid Dynamics, The Netherlands
}

\begin{abstract}
A comprehensive computational model for simulating magnetic drug targeting was developed and extensively tested in a cylindrical geometry. The efficiency for particle capture in a specific magnetic field and geometry was shown to be dependent on a single dimensionless number. The effect of secondary flows, a non-Newtonian viscosity and oscillatory flows were quantified. Simulations under the demanding flow conditions of the left coronary artery were performed. Using the properties of present-day magnetic carriers and superconducting magnets, approximately one third of $4 \mu \mathrm{m}$ particles could be captured with an external field. These promising results could open up the way to a minimally invasive treatment of coronary atherosclerosis.
\end{abstract}

Keywords - Magnetic Drug Targeting (MDT), Coronary Artery, Particle Capture, Inhomogeneous Magnetic Fields, Atherosclerosis

\section{INTRODUCTION}

The targeting of drugs to a specific location inside the human body can be significantly enhanced using magnetic fields. Drugs attached to a magnetic particle can be slowed down or even captured from the bloodstream in the presence of a non-uniform magnetic field. This promising magnetic drug targeting (MDT) technique for improving the specificity of for example chemotherapy has recently been successfully applied to human patients. We believe that numerical simulations can form a valuable tool for optimizing and estimating in advance the effectiveness of a treatment. Our goal is to be able to perform such simulations on a patient-specific basis, this paper reporting some of the recent developments in that direction.

\section{THEORY}

\section{A. Magnetization Force}

Various materials acquire a net magnetic dipole moment when placed in an external magnetic field, in reaction to which a force is exerted on the material given by

$$
\vec{F}_{M}=\mu_{0} V \vec{M} \cdot \nabla \vec{H}
$$

with $V$ the volume of the material, $\mu_{0}=4 \pi \cdot 10^{-7} \mathrm{~N} / A^{2}$ the magnetic permeability of vacuum, $\vec{M}$ the magnetization (magnetic dipole moment per unit volume) and $\vec{H}$ the applied (auxiliary) magnetic field. For many materials the magnetization can be taken approximately proportional to the applied magnetic field up to a certain value. Beyond this saturation magnetization $\vec{M}_{\text {sat }}$ all the constituent dipoles are aligned with the field and no further increase in magnetization is possible

$$
\vec{M}= \begin{cases}\chi \vec{H} & H<M_{\text {sat }} / \chi \\ \vec{M}_{\text {sat }} & H \geq M_{\text {sat }} / \chi\end{cases}
$$

For fully oxygenated blood the proportionality constant, the magnetic susceptibility, is approximately $-6.6 \cdot 10^{-7}$. This small and negative number implies via Eqn. 1 a slight repulsive force from inhomogeneous magnetic fields.

\section{B. Equations of motion}

For the blood velocity $\vec{u}$ the Navier-Stokes equations for an incompressible fluid $(\nabla \cdot \vec{u}=0)$ are solved augmented with the magnetization force

$$
\rho\left(\frac{\partial \vec{u}}{\partial t}+\vec{u} \cdot \nabla \vec{u}\right)=-\nabla p+\eta \nabla^{2} \vec{u}+\vec{F}_{M} / V
$$

The relative importance of the inertial forces compared to the viscous forces is denoted by the dimensionless Reynolds number $R e=\rho v l / \eta$ with $v$ and $l$ characteristic velocity and length scales of the flow under consideration.

The particle trajectories $\vec{r}(t)$ and velocities $\vec{u}_{p}=d \vec{r}(t) / d t$ of particles with mass $m$ can be obtained from

$$
m \frac{d^{2} \vec{r}(t)}{d t^{2}}=\vec{F}_{D}+\vec{F}_{M}
$$


For small particle Reynolds numbers (with $v=\left|\vec{u}-\vec{u}_{p}\right|$ and $l=D$ the particle diameter) the drag force $\vec{F}_{D}=3 \pi \eta\left(\vec{u}-\vec{u}_{p}\right) D$ and usually the particle acceleration is negligible such that $\vec{F}_{D}+\vec{F}_{M}=0$. This balance between magnetization force and drag force shows that the relevant dimensionless quantity to be maximized for particle capture is

$$
M n_{p} \equiv \frac{F_{M}}{F_{D}}=\frac{\left|\mu_{0} V \vec{M} \cdot \nabla \vec{H}\right|}{3 \pi \eta \vec{u} D}=\frac{\mu_{0} D^{2}|\vec{M} \cdot \nabla \vec{H}|}{18 \eta u}
$$

This shows for example that for twice as large particles the flow velocity can be four times as high, or the field gradient four times as small to capture the same amount of particles.

\section{MEthods}

The fluid and particle equations were solved in Fluent 6.3 software by ANSYS, Inc. which is a linear multigrid finite volume solver. Eqn. 3 is solved fully implicit with a quadratic upwind (QUICK) discretization of the nonlinear term. Various used-defined functions were written in $\mathrm{C}$ to increase the functionality of material properties, boundary conditions and external forces.

Eqn. 4 is numerically integrated using a sixth order Runge-Kutta scheme whenever the first term is significant and an implicit scheme otherwise. Alternative schemes, various time steps and accuracy options were tested with no appreciable differences, providing confidence that the integration is performed accurately. To validate the implementation a comparison was made with an analytical result for which satisfactory correspondence was obtained.

For the complex geometry of the left coronary artery a mesh obtained from average data of the angiographies of 83 healthy patients [1] is used.

\section{RESULTS}

\section{A. Cylindrical geometry}

Fluid magnetization: Due to its negative magnetic susceptibility, oxygenated blood experiences a magnetization force opposing an existing magnetic field gradient. Simulations in the cylindrical geometry of Fig. 1 showed that when an infinitely long current carrying wire was placed halfway, fluid motion perpendicular to the main flow was induced as shown in Fig. 2. These secondary flow patterns arise as fluid is pushed away in axial direction,

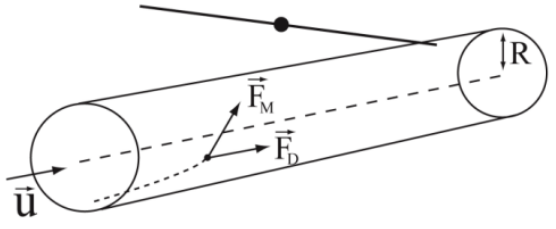

Fig. 1 A schematic overview of the used geometry. The line with dot indicates the location of a current carrying wire. The magnetization force and drag force on a particle are indicated. A tube length of $7 \mathrm{~cm}$ (larger

for high $\mathrm{Re}$ ) and a diameter of $7 \mathrm{~mm}$ have been used throughout.

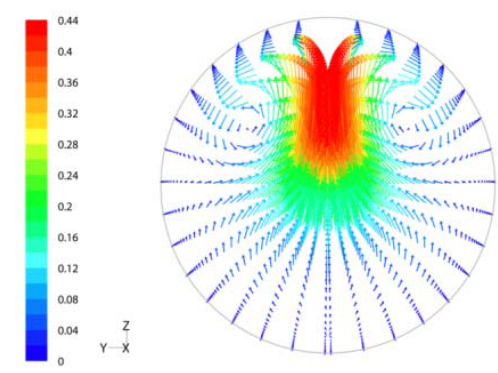

Fig. 2 Secondary motions induced by the magnetization force of a wire carrying a current of $10^{5} \mathrm{~A}$, placed at a distance of $1 \mathrm{~cm}$ from the cylinder axis. The coloring indicates the secondary flow velocity as a fraction of the average main velocity of $0.1 \mathrm{~mm} / \mathrm{s}(\mathrm{Re}=0.2)$.

fluid is drawn in by continuity below the wire where an opposing pressure gradient arises.

In order to investigate the importance of these secondary motions for magnetic drug targeting, particles were inserted homogeneously distributed over the circular cross-section of the domain. It was found that the overall capture efficiency ((in-out)/in) decreased due to the downward fluid motion along the tube wall dragging especially the smaller, less attracted particles away from the magnet. The effect was found to be fairly small though and dependent on the particle size. Even when the secondary flow became of the same order as the main flow the capture efficiency was influenced by typically only $15 \%$.

The strength of the secondary motions was found to depend mainly on the strength of the magnetization force relative to inertial forces, and thus for a given magnetic field on the Reynolds number. Only for physiologically low Reynolds numbers do these secondary flows become of the some order of magnitude as the main flow.

Non-Newtonian viscosity: The viscosity influences the drag force on a particle directly, but also through its effect

Table 1 Material properties used for cylindrical geometry

\begin{tabular}{lll}
\hline \multicolumn{1}{c}{ Material properties } & \multicolumn{1}{c}{ Blood } & \multicolumn{1}{c}{ Particles } \\
\hline Density $\left(\mathrm{kg} / \mathrm{m}^{3}\right)$ & 1000 & 5150 \\
Magnetic susceptibility (-) & $-6.6 \cdot 10^{-7}$ & 3 \\
\hline
\end{tabular}




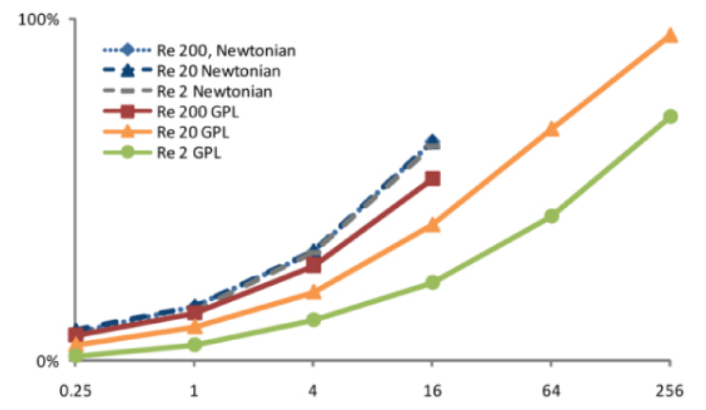

Fig. 3 The capture efficiency as a function of $\mathrm{Mn}_{\mathrm{p}}$ for blood flow in a cylindrical pipe using a GPL viscosity model [2] compared to a Newtonian model for various Reynolds numbers.

on the flow field. Blood has a higher viscosity for smaller shear rates existing e.g. near the centerline of a straight pipe flow. Accordingly the flow profile becomes flattened compared to the parabolic profile arising for a constant viscosity. This flattening is most pronounced for low Reynolds numbers and a decrease in centerline velocity by over $10 \%$ for Reynolds numbers below 200 was obtained using the generalized power law (GPL) model of [2].

In order to be able to compare the effect on the capture of particles for various Reynolds numbers we used different particle sizes such that the quantity $M n_{p}$ (evaluated using the average flow velocity and the field gradient at the centerline below the wire) was equal in all simulations. So for ten times higher Reynolds numbers we used $\sqrt{10}$ times larger particles.

From Fig. 3 we see that for a Newtonian viscosity model (with $\eta=3.5 \cdot 10^{-3}$ Pas, as used up to now) the capture efficiency curves for all three simulated Reynolds numbers almost perfectly overlap. So using a two times larger particle diameter and at the same time a four times higher flow velocity in this case yields exactly the same capture efficiency. This implies that the characterization of the capture efficiency in terms of $\mathrm{Mn}_{\mathrm{p}}$ is a very good one. Equivalently one can conclude that particle inertia is indeed negligible in this case. We also see however that using the same constant viscosity for $\mathrm{Mn}_{\mathrm{p}}$ does not suffice and that fewer particles are captured due to an increased viscosity at lower Reynolds numbers.

Oscillatory flow: To investigate what impact oscillatory flow has on the magnetic capturing of particles a superposition of a steady and a harmonically oscillating flow of equal amplitude was used. For the inlet velocity the analytical solution that exists for unsteady periodic cylindrical pipe flow was used. Periods of $5.5 \mathrm{~s}, 1.375 \mathrm{~s}$ and $0.34375 \mathrm{~s}$ were used to yield Womersley numbers of $\alpha \equiv R \sqrt{2 \pi \rho / \eta T}=2,4$ and 8 representative for small arteries to very large arteries respectively.

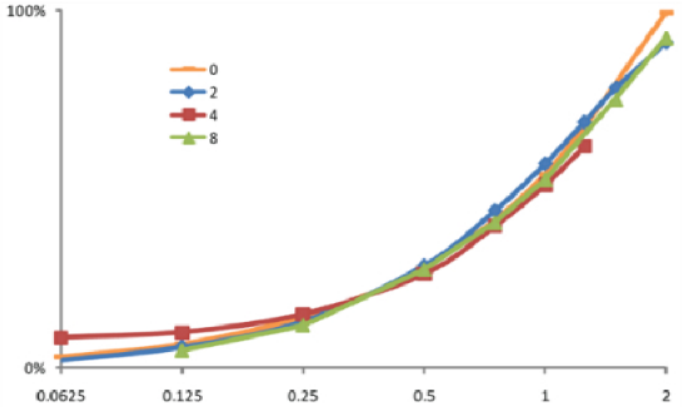

Fig. 4 The capture efficiency vs. particle diameter $(\mu \mathrm{m})$ for various value of the Womersley parameter $\alpha$, zero being a steady flow.

It was found that depending on the time of injection less or more particles can be captured compared to steady flow conditions, but that averaged over one cycle these differences are not significant (see Fig. 4). A similar simulation with elastic cylinder walls extending up to $5 \%$ radially at the highest pressure led to similar results.

Although these conclusions might not hold in more complex geometries, they do show that the specifics of the transient flow profile on the magnetic capturing of particles might not be of crucial importance compared to the average flow.

\section{B. Left Coronary Artery}

We next test the capabilities of the magnetic drug targeting technique under the demanding flow conditions of a left coronary artery using present-day available magnetic fields and materials. We use state-of-the-art cylindrical superconducting magnets [4] in which a supercurrent flows primarily near the surface. Owing to this physical origin a good fit to the field is obtained using that of a circular line current (diameter $4.5 \mathrm{~cm}$ ) halfway the magnet (i.e. $7.5 \mathrm{~mm}$ below its surface) for a current of $2.1 \cdot 10^{5} \mathrm{~A}$ (see Fig. 5).

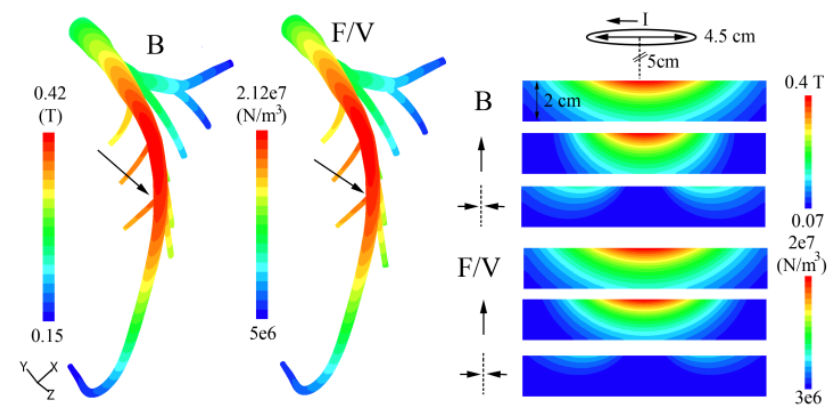

Fig. 5 The magnetic field $\mathrm{B}(\mathrm{T})$ and magnetization force per unit volume $\mathrm{F} / \mathrm{V}(\mathrm{N} / \mathrm{m} 3)$. The magnitude, vertical and radial components 5 to $7 \mathrm{~cm}$ below the circular current loop (right) and the magnitudes at the location of the coronary artery (left) are displayed. 

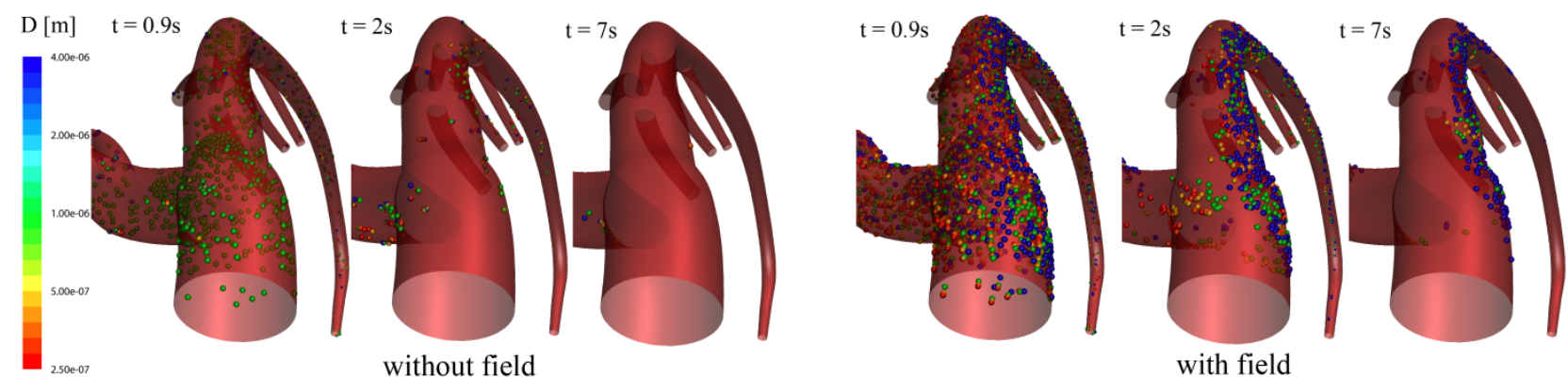

Fig. 6 The distribution of particles in seconds after the first injection of particles as seen from the inlet. In the absence of a magnetic field the particles of different diameter overlap as they follow approximately the same path due to negligible inertia. In the case of an applied magnetic field especially the heavier particles are attracted towards the sidewalls where they can exchange any attached drugs with the arterial wall.
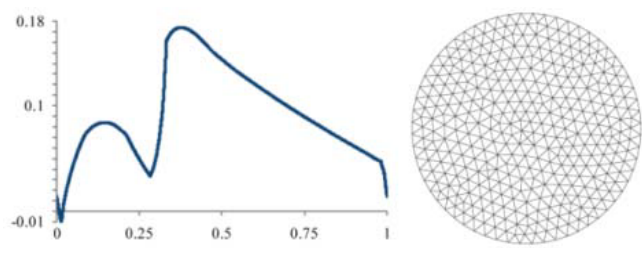

Fig. 7 The inlet velocity $\bar{u}(t) \cdot(\mathrm{m} / \mathrm{s})$ vs. time (s) and mesh. A somewhat flattened inlet velocity profile $u(r)=(4 / 3) \bar{u}(t)\left(1-(r / R)^{6}\right)$ was used

For the properties of the particles we resorted to the high susceptibility, high saturation magnetization material iron which has been successfully made into drug susceptible carriers using carbon coatings [4]. With 67.5 weight $\%$ iron the particles have a density of approximately $6450 \mathrm{~kg} / \mathrm{m}^{3}$ and a saturation magnetization $10^{6} \mathrm{~A} / \mathrm{m}$. As the particles are almost completely saturated even for fields as low as $0.05 \mathrm{~T}$ they are assumed saturated throughout the calculation. Particles with diameters of 250nm, 500nm, $1 \mu \mathrm{m}, 2 \mu \mathrm{m}$ and $4 \mu \mathrm{m}$ were inserted homogeneously distributed over the inlet and spread over one flow cycle of $1 \mathrm{~s}$ at consecutive intervals of $0.1 \mathrm{~s}$. The particle velocity was assumed to vanish after impact with the vessel wall.

The cylindrical superconducting magnet was assumed to be positioned approximately at the patient's chest (its center at $5 \mathrm{~cm}$ from the vessel), directed towards the outer curvature of the left main coronary artery (the arrow in Fig. 5). The region opposite flow dividers is a well-known location of low endothelial shear stress correlated with the formation of atheromatous plaques. A possible future application could include loading the carbon-coated particles with thrombolytic agents to prevent a threatening plaque rupture.

The results are summarized in Fig. 6 where the effect of the magnetic field can be clearly seen from the distribution of primarily the largest particles near the arterial wall. A significant fraction of $34 \%$ of the $4 \mu \mathrm{m}$ particles could be captured, with this efficiency rapidly decreasing for smaller diameters. Note that $\mathrm{Mn}_{\mathrm{p}}$ evaluated at the target location is approximately $1 / 20$ for $\mathrm{D}=4 \mu \mathrm{m}$, but still many particles can be captured owing to the wide extent of the magnetic field.

\section{Conclusions}

The efficiency for magnetic particle capture was for a given geometry and magnetic field found to be effectively described by a single dimensionless number representing the ratio between the magnetization and the drag force. A higher viscosity however should be used for smaller strains to take into account the non-Newtonian behavior of blood. Secondary and oscillatory flows were found to be of little importance. Simulations of the left coronary artery showed that present day materials can be used to magnetically capture a significant fraction of $4 \mu \mathrm{m}$ particles using external fields. These results could lead the way to a minimally invasive treatment of coronary atherosclerosis.

\section{AcKnowledgment}

Much gratitude goes out to Johannes V. Soulis (Demokrition University of Thrace, Greece) for providing the left coronary artery mesh and pulsatile flow profile.

\section{REFERENCES}

1. Giannoglou GD, Soulis JV, Farmakis TM, Louridas GE (2003) Molecular Viscosity Distribution in the Left Coronary Artery Tree. Comp in Cardiol 30:641-644

2. Johnston BM, Johnston PR, Corney S, Kilpatrick, D (2003) NonNewtonian blood flow in human right coronary arteries: steady state simulations. J Biomech 37:709-720 
3. Takeda S-i, Mishima F, Fujimoto S et al. (2007) Development of magnetically targeted drug delivery system using superconducting magnet. J Magn Magn Mat 311:367-371

4. Cao H, Huang G, Xuan S, Wu Q et al. (2008) Synthesis and characterization of carbon-coated iron core/shell nanostructures. J. Alloys Comp 448:272-276
Author: J.W. (Willem) Haverkort

Institute: Delft University of Technology,

Department of Multi-Scale Physics

Street: Prins Bernhardlaan 6

City: Delft

Country: The Netherlands

Email: willemhaverkort@gmail.com 